\title{
The Misdiagnosis and Consequences of the Odontogenic Orocutaneous Fistula by Medical Doctors: A Case Report
}

\author{
(1) Nihal Altunok Ünlü, (1) Halenur Altan \\ Tokat Gaziosmanpaşa University Faculty of Dentistry, Department of Pediatric Dentistry, Tokat, Turkey
}

\begin{abstract}
It is possible to describe the term fistula as an abnormal connection between different parts of the body. Untreated, specific infections or diseases, chronic infections, congenital deformities, traumatic injuries and postoperative recovery abnormalities may lead to the formation of fistulas. The most common types of oral fistulas can be classified as dentoalveolar, oroantral, oronasal, and orocutaneous fistulas.

Dentists and doctors can frequently misdiagnose odontogenic cutaneous fistulas as cutaneous lesions or non-odontogenic infections. As a consequence of an incorrect diagnosis, it is possible for patients to undergo unneeded and ineffective treatments, such as the surgical excision of the cutaneous lesion, multiple biopsies, and repeated antibiotic administration. It has been observed that misevaluation of the lesion and repeated ineffective interventions can lead to scar formation on the skin, pit defects, hyperpigmentation, and iatrophobia.

In this case report, we aimed to present the follow-up results of a patient with an odontogenic orocutaneous fistula who was misdiagnosed and treated by medical doctors from different branches.
\end{abstract}

Keywords: Odontogenic cutaneous fistula, endodontic treatment, focal infection

\section{Introduction}

It is possible to describe the term fistula as an abnormal connection between different parts of the body. They can be congenital or acquired and also they can also develop in different parts of the body. Specific untreated infections or diseases, chronic infections, congenital deformities, traumatic injuries and postoperative recovery abnormalities may lead to the formation of fistulas. Despite the fact that oronasal, orocutaneous, oroantral and dentoalveolar fistulas are the most frequently observed types because of oral cavity, it is possible for an oral fistula to be dependent on the origin (1).
A pathological pathway between the oral cavity and alveolar bone is known as a dentoalveolar fistula, which generally arises from infected cysts, necrotic teeth, periodontal inflammation, trauma or mandibular or maxillary fractures (2). In the case of a necrotic dental pulp, the root canal develops into a potential site of bacterial colonization. In this case, if it is not treated, the infection spreads into the periradicular area, which leads to apical periodontitis, and follows the path of least resistance in the bone and soft tissue. The direction and site of the fistula to the surface are determined by the location of muscle attachments and the position of root tips. Following the spread of the periradicular infection and the disappearance

*This case report was presented as an oral presentation at the International Dentistry Congress of Erciyes University on 26-29 February 2020. 
of the cortical wall of the alveolar bone, the fistula follows interstitial spaces. Periradicular infections can reach the oral mucosa or the skin and induce the formation of fistula despite the fact that most of them remain limited within the loose connective tissue compartments and thus lead to the formation of abscess (1).

It is possible to perform a diagnosis by radiographic examination, dental examination, radiologic evaluation, or by placing gutta-percha or similar radiopaque material in the fistula tract. The evidence of the radiolucent periapical disease process is shown by dental periapical or panoramic radiographic images. It is necessary for patients to be assessed by orthopantomography, and if possible, by conebeam computed tomography. From histological aspects, the cutaneous fistula tract is generally composed of epithelium or granulomatous tissue. Antibiotic therapy, biopsies and multiple surgical excision might be carried out due to diagnostic errors (3). A carious tooth or a history of previous trauma can help diagnose in intraoral examination while evaluating these patients. Nevertheless, dermal lesions are not specific and can also be observed for abscesses, ulcers, cysts and scars (4).

In this case report, we aim to present the dental diagnosis, treatment, and follow-up of a patient with an odontogenic orocutaneous fistula who was misdiagnosed and subsequently attempts were made to treat the patient by medical doctors from different branches.

\section{Case Report}

An 11-year-old male patient applied to pediatric dentistry clinic with a complaint of a non-healing wound on his right lower jaw. As a result of the anamnesis taken from the patient, it was determined that he did not have any systemic disease and had undergone three drainage operations on his right lower jaw in the previous year. It was ascertained that he had applied to a paediatric surgery department six months before applying to our clinic, due to periodic discharge under the right chin and redness. He was diagnosed with sebaceous gland inflammation by a paediatric surgeon. The patient was operated on twice by the paediatric surgeon, and it was ascertained that the patient was then referred to a plastic surgeon six months later because of a lack of improvement in the site and aesthetic concerns. The patient was operated on by the plastic surgeon with the diagnosis of sebaceous gland inflammation. Subsequently, the patient was referred to our clinic after the plastic surgeon observed that the discharge was associated with the jaw bone during the operation.
In the clinical examination, the patient had an erythematous extraoral fistula approximately $1 \mathrm{~cm}$ in diameter in the lower right area with sutures around it. In tooth 46 of the patient who was thought to have extraoral fistula, a mismatch was observed between the composite resin restoration, cavity wall, and restoration material. Percussion sensitivity was minimum in tooth 46, and intraoral swelling and redness were not observed. In a radiographic examination, an in-depth restoration material and a large periapical lesion were identified in tooth 46.

Root canal treatment was started in the same session in our clinic. Calcium hydroxide dressing was applied and it was filled with temporary restoration material. Two sessions of calcium hydroxide dressing were carried out at two-week intervals. When improvement in the fistula tract was observed in the fourth session, treatment was terminated by filling and root canal treatment (Figure 1).

In the first month check-up of the patient after root canal treatment, a hard, nodular structure was noticeable during palpation at the borders of the site of the surgical operation. The patient was referred to a paediatric unit. As a result of paediatrics consultation, it was determined that there was scar tissue and injections with cortisone content were made in this region. Small plaques of white colour were observed in the scar tissue (Figure 2). The patient was then referred to a plastic surgeon to assess treatment in terms of aesthetic appearance.

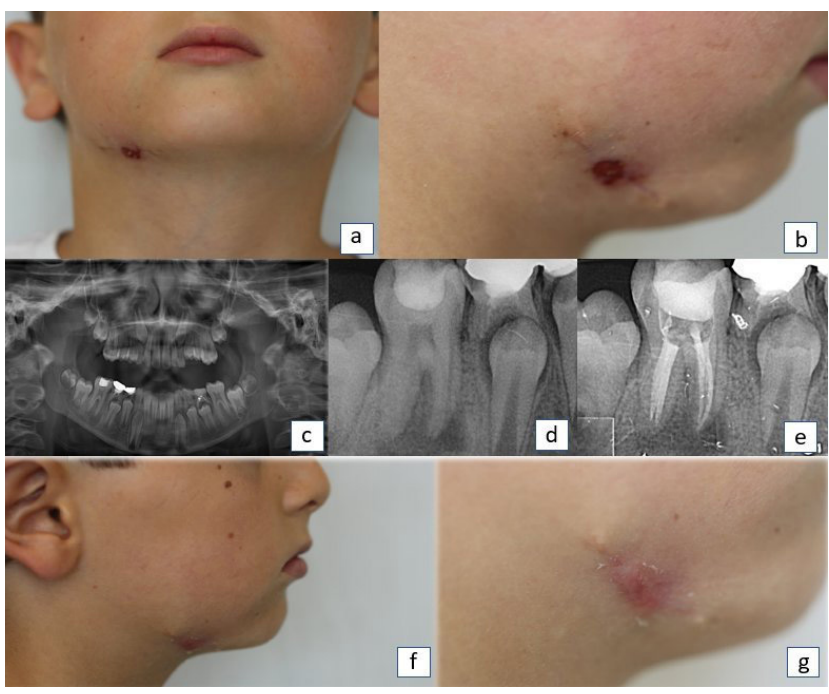

Figure 1. a, b) Preoperative extraoral photograph. c) Preoperative panoramic radiograph of the patient. d) Preoperative periapical radiograph of the patient. e) Postoperative periapical radiograph. f, g) Postoperative extraoral photography 


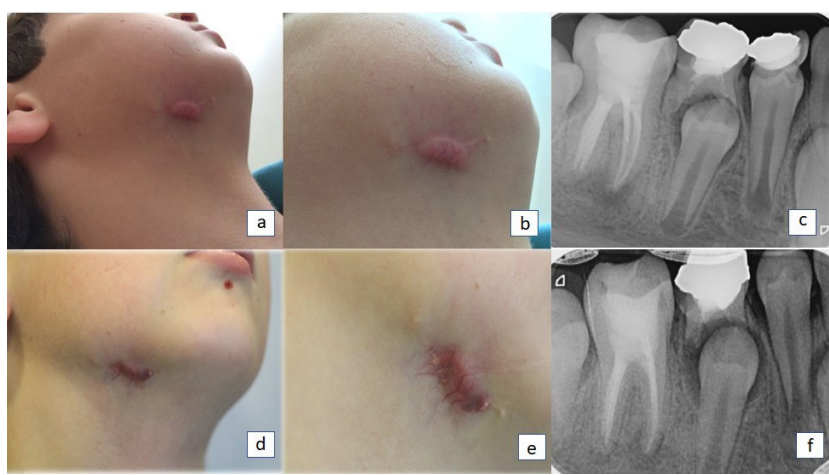

Figure 2. a, b) Extraoral photography of in the first month check-up of the patient after root canal treatment. c) Periapical radiography of in the first month check-up of the patient after root canal treatment. $d$, e) Extraoral photography of in the third month check-up of the patient after root canal treatment. f) Periapical radiography of in the third month check-up of the patient after root canal treatment

\section{Discussion}

The evaluation of the cutaneous fistula tract should begin with a comprehensive history and the awareness that any cutaneous lesion of the face and neck may be of dental origin (5). Patients may complain about dental problems. However, patients may not remember an acute or painful story of onset. With the persistence of a cutaneous lesion, there may also be episodic bleeding or drainage complaints from the cutaneous area. For the correct diagnosis of cutaneous sinus of dental origin, the external appearance of the lesion should be addressed. The most characteristic feature of this type of nodule is depression or withdrawal below the normal surface. This cutaneous retraction or cavitation is caused by the fixation of the fistula to the underlying tissues and may be secondary to the healing process or a late finding inactive disease. Previously biopsied or treated lesions are usually characterized by the absence of at least a portion of the nodule and often with a fistula mouth at the base of the fixed depression (6).

Non-surgical endodontic treatment is often the preferred treatment if the tooth can be restored in cases diagnosed as odontogenic. Extraction is indicated for teeth that cannot be restored (7). The failure of the cutaneous fistula tract to heal after adequate root canal treatment or extraction requires further evaluation, microbiological sampling, and biopsy. In this case report, an 11-year-old paediatric patient was diagnosed with odontogenic orocutaneous fistula of tooth 36 origins, and an improvement was observed only with endodontic treatment. When the age of the patient was considered, it was essential to achieve successful results with endodontic treatment without the need for complex surgical interventions. Surgical operations which were initially performed by medical doctors led to negative aesthetic and psychological results in this paediatric patient.

Systemic antibiotic administration is not recommended in patients with high immune resistance, no systemic signs or symptoms, no prophylactic antibiotic requirement, and with cutaneous fistula tract. The fistula tract provides the drainage of the primary odontogenic area and prevents swelling and pain caused by pressure.

Following a thorough cleaning of the root canal system, the disappearance of the sinus tract generally takes 5 to 14 days (8). From a histological aspect, granulomatous tissue generally covers these tracts. Granulation allows for their healing following the removal of the root canal infection. Occasionally, a shrunken, hyperpigmented, or pink scar is caused by the healing of the fistula tract (8). Hypertrophic scars and keloids are formed as a result of uncontrolled proliferation of fibrous tissue resulting from a deviation in the normal wound healing process following an injury to the skin. Scars can be classified as mature scars, immature scars, keloid scars, hypertrophic scars, and contracture scars if healing results in cutaneous retraction or cavitation. Treatment with cosmetic surgery may be required at a later date. In the case of scar formation, cortisone can be administered. Depending on the administration of cortisone, the storage of the drug under the skin may lead to small white-coloured plaques in the scar tissue. Linear hypertrophic scar formation was observed in this case report. Coloured white plaques were found as a result of cortisone treatment.

The clinician who is to conduct any treatment should consider the possibility of dental origin while diagnosing a cutaneous fistula tract. Consultation based on cooperation is required among doctors (primarily dermatologists), surgeons, and dentists for a comprehensive diagnosis. By means of the identification of the correct nature of the lesion, rapid treatment is simplified, aesthetic problems and patient discomfort are minimized, and the likelihood of further complications is significantly decreased.

It is of critical importance for medical doctors to refer those patients who present with complaints of head and neck abscesses, fistulas, and pain to a dentist with suspected odontogenic infection. Those patients referred to the dentist can be treated in a short time with endodontic root canal treatment or tooth extraction. It was observed that misevaluation of the lesion and repeated ineffective interventions in this case study led to scar formation on the skin, pit defects, hyperpigmentation, and iatrophobia. 


\section{Ethics}

Informed Consent: The informed consent form was obtained from the parent before the treatment.

Peer-review: Externally peer-reviewed.

\section{Authorship Contributions}

Surgical and Medical Practices: N.A.Ü., Concept: H.A., Design: H.A., Data Collection or Processing: N.A.Ü., Analysis or Interpretation: H.A., Literature Search: N.A.Ü., Writing: N.A.Ü.,

Conflict of Interest: No conflict of interest was declared by the authors.

Financial Disclosure: The authors declared that this study received no financial support.

\section{References}

1. Cansiz E, Gultekin A, Koltuk M, Cakarer S. Treatment of oral fistulas. In: Kalantar Motamedi MH (ed). A Textbook of Advanced Oral and Maxillofacial Surgery. London, Intechopen, 2016.
2. Assery M, Al Shamrani S. Cutaneous facial sinus tract of dental origin: a clinical case report. Saudi Dental 2001; 13:37-9.

3. Giménez-García R, Martinez-Vera F, Fuentes-Vera L. Cutaneous sinus tracts of odontogenic origin: two case reports. I Am Board Fam Med 2015; 28:838-40.

4. Tian J, Liang G, Qi W, Jiang H. Odontogenic cutaneous sinus tract associated with a mandibular second molar having a rare distolingual root: a case report. Head Face Med 2015; 17:13.

5. Cohen PR, Eliezri YD. Cutaneous odontogenic sinus simulating a basal cell carcinoma: case report and literature review. Plast Reconstr Surg 1990; 86:123-7.

6. Stoll HL, Solomon HA. Cutaneous sinuses of dental origin. JAMA 1963; 184:120-4.

7. Gupta M, Das D, Kapur R, Sibal N. A clinical predicament diagnosis and differential diagnosis of cutaneous facial sinus tracts of dental origin: a series of case reports. Oral Surg Oral Med Oral Pathol Oral Radiol Endod 2011; 112:e132-6. doi: 10.1016/j.tripleo.2011.05.037.

8. Spear KL, Sheridan PJ, Perry HO. Sinus tracts to the chin and jaw of dental origin. I Am Acad Dermatol 1983; 8:486-92. 